\title{
The Effect of Polyurethane Production Temperature on its Oil Absorbtion Capacity
}

\author{
Petr V. Zgonnik, Tamara S. Shakhparonova and Vladislav A. Chernyaev \\ Department of General and Physical Chemistry, National Mineral Resources University \\ (Mining University), 2, 21st Line, Saint Petersburg, 199106, Russian Federation. \\ http://dx.doi.org/10.13005/bbra/2063
}

(Received: 21 December 2015; accepted: 10 February 2016)

\begin{abstract}
The paper studies oil absorption by polyurethane sorbents synthesized at temperatures of $20,30,40,50,60$ and $70^{\circ} \mathrm{C}$ that is necessary for the development of physicochemical foundations of the oil absorbents synthesis. It is revealed that the kinetic curves of oil absorption of the obtained sorbents, as well as previously studied industrial polyurethane foams existing in a glassy state, are characterized by the presence of maximum (at $30 \mathrm{~min}$ ), while increasing the synthesis temperature leads to an increase in oil absorption. The author gives interpretation of obtained patterns, which are confirmed, in particular, by the electron-microscopic study.
\end{abstract}

Key words: Polyurethane foam, process temperature, process activation energy, diffusion of the blowing agent.

One of the global problems facing humanity is the need to avoid environmental degradation in consequence of natural resources development and conversion of natural systems. It is known that the oil and oil products are one of the most harmful classes of pollutants (Kogan et al., 2014). Today Russia is one of the world leaders in oil production.

The elimination of accidental spills of oil, oil products and organic substances in soil and water is one of the most challenging tasks in the field of environmental protection. Annually, about $10 \mathrm{mln}$ tons of crude oil and petroleum products from different sources get to the global ocean (1 tone of oil spreading over the water surface forms a film with the thickness ranging from micrometers to $2 \mathrm{~cm}$ and covering an area of about $12 \mathrm{~km}^{2}$ ). Russia ranks second in oil production among oil-

\footnotetext{
* To whom all correspondence should be addressed.
}

producing countries. It produces $12.3 \%$ of all world oil reserves (Saudi Arabia produces 12.7\%). According to the "Interfax" agency, the volumes of oil and gas condensate extractions in Russia in 2014 increased by $0.7 \%$ as compared to the same period of 2013 and amounted to $526,753 \mathrm{mln}$ tons (10,578 mln barrels per day). However, the structure of oil spills and environmental pollution in Russia has its own specifics. Russia is a continental country, and the main quantity of the produced oil is transported by land. The main part of Russian oil is produced in Siberia (in the Tyumen, Tomsk and Irkutsk regions). Oil is pumped through pipelines to various regions of Russia and neighboring countries that relatively recently were part of the Soviet Union, as well as to maritime terminals and countries of the European Union. These pipelines are laid under the ground and under water. Often, especially under water, the pipes are cracked due to the corrosion and the oil starts to seep out. In just two years, from 2002 to 2004, in Russia there were 65 serious accidents on oil and oil products pipelines. At that, the hydrocarbons 
get into the soil and waterways, causing damage to the flora and fauna of reservoirs. According to official statistics, accidental spills result in lost up to $1 \mathrm{mln}$ tons of oil per year. These data are incomplete. According to "Greenpeace" estimates, in Russia oil losses during production and transportation amount to about $1 \%$. For example, non-commercial partnership "Ecology Center of Fuel-energy Complex" estimates the losses of oilwithin the range of 3.5-4.5\%. Accordingly, in recent years, at oil production at the level of about $500 \mathrm{mln}$ tons per year, the amount of spilled oil ranges from 18 to $23 \mathrm{mln}$ tons annually, or in money terms, from 14.2 to 17.2 bln rubles.

We should note some peculiarities of the Russian legislation, which complicate registration, accounting for and responding to emergencies arising in the course of oil production and transportation. Very complicated procedure, limited by law, does not allow reacting promptly to violations. Upon receipt of information about the oil spill by the Federal Supervisory Natural Resources Management Service (Rosprirodnadzor), the information is carefully checked. If the violations did occur, verification documents are submitted to the Federal Subsoil Resources Management Agency (Rosnedra). Besides, a special Commission is appointed which first develops measures to remedy the situation, and then gives the subsurface user certain time to eliminate the detected violations. At the current level of interaction between different agencies in Russia, it is easy to imagine what we get as a result. Despite the fact that today the Russian ecological laws are the most stringent in the world, there are many loopholes it the law that is well known by oil companies, which easily avoid their responsibility. For example, at present all oil spills are divided into two categories - pre-emergency and emergency spills. The spill of less than 10 cubic meters of oil is considered pre-emergency. It's amazing that in case of pre-emergency situation companies do not have to pay even penalties. The situation is complicated also by the fact that it is impossible to determine, at least on land, how much oil pours out. No one keeps records of oil spills, and the most importantly - nobody evaluates the quantity of oil spilled. State regulatory body, Federal Supervisory Natural Resources Management Service (Rosprirodnadzor) collects data provided by the organizations and extractive industry companies in relation to such incidents and mitigations of their consequences. However, according to environmental NGOs, these data are not objective since the indicated figures are heavily underestimated. Companies do not want to pay compensation and seek to reduce the number of emergences or eliminate consequences of oil spills just partially, for example, just in the area of the burst pipes, i.e. exclusively within the field of view of inspecting organizations. The most frequently unchecked oil spills occur in a vast and sparsely populated expanse of tundra and Northern taiga. The bulk of oil spills occur during its transportation through pipelines. Most regions, where oil is extracted, are characterized by harsh climatic conditions, where the winter temperature falls to 40 degrees below zero. At such low temperatures, the tube metal becomes brittle, and the tubes are often damaged against the background of irregular inspections and lack of periodic replacement of the pipes. In Russia, the length of just state pipelines is more than 70 thousand $\mathrm{km}$. It is difficult to calculate the length of the non-governmental pipelines, but it is safe to say that it greatly exceeds the "state" part. Only in Eastern Siberia the length of local pipelines connecting various fields is more than 100 thousand kilometers. It is exactly these pipelines that are involved in the majority of emergences. The most common cause of accident (about 90\%) is destruction of pipes due to corrosion and wear. One of the last major accidents on the oil pipeline occurred in 2011 at the Fedorovskoye field in the area of Surgut. The oil gusher hit at a height of 10 meters over two days. Emergency crews had to pump out over 4,000 cubic meters of spilled oil. At the same time, regional management of Rosprirodnadzor assessed the damage to the extent of $7 \mathrm{mln}$ rubles. Earlier, on May 17, 2005 on the same field there was an accident at one of the wells, which resulted in an oil gusher $12 \mathrm{~m}$ in height.

Mechanical damage to the pipeline is another common cause of serious accidents. Most of them are caused by so-called illegal tie-ins, when criminals are trying to steal oil from the state or a private company and then to produce oil products onside the controlled territory. At that, most illegal tie-ins are found on the pipelines pumping petroleum products. Obviously, illegal seizure of 
quality petroleum products, fuels and lubricating oils is more profitable than crude oil. However, one should not think that mechanical damage to pipelines can be caused only by action of the human factor. Thus in 1995, during the earthquake on Sakhalin, many pipelines were immediately destroyed that in accordance to the official data has led to spillage of at least 6 thousand tons of oil. It should be noted that in accordance with the available official estimates at least $10-15 \%$ of all oil lost due to leakage fall into the Russian rivers and then are carried into the global oceans.

For the time being, none of the spills that occurred in Russia have approached in terms of their scope to the unprecedented accident occurred on the deepwater oil platform of BP company, which happaned on April 20, 2010 in the Gulf of Mexico. Then nearly $5 \mathrm{mln}$ barrels of oil (794 900 cubic meters) spilled in the Mexican Gulf. At that, the area of oil spill amounted to 75 thousand sq. $\mathrm{km}$. The largest accident in Russia, coupled with the pollution of water surface by oil, occurred on November 11, 2007, when the "Volgoneft-139" tanker was broken by wave during a storm in the Kerch Strait, and more than 2 thousand tons of fuel oil spilled into the sea. The damage caused to environment was estimated in the amount of 10.5 billion rubles. However, in the near future abovedescribed ratio between the leakages during the oil transportation over land and oil pollutions of water objects must change since Russia has embarked on several projects of oil production on the shelf that will lead to significant increase in proportion of emergences related to contamination of the aquatic environment.

Based on the foregoing, we can predict that in the future, in the wake of rising crude oil output on the shelf and increasing the proportion of waterway transportation of the oil and oil products, the pollution of reservoirs by oil will increase (Kogan et al., 2014). During production, storage and transportation of crude oil it is impossible to prevent completely the risk of accidents associated with spills of oil and oil products into the environment. The most devastating accidents are those associated with transportation of oil by tankers on the waterways (Praba Karan et al., 2011).

To eliminate pollution of water surface by oil and oil products, various methods of physical chemistry, based in particular, on adsorption, are used widely and quite successfully. For the most effective and affordable solution of the considered problem, a number of adsorbents of different nature are used. Natural inorganic adsorbents such as clays (Carmody et al., 2007), perlite (Bastani et al., 2006), modified perlite (Qi et al., 2013) as well as glass wool (Annunciado et al. 2005) are quite inexpensive and affordable. Also there are adsorbents developed based on natural organic matter such as hay, feather, straw, and peat moss (Suni et al., 2004). Natural adsorbents have low adsorption capacity, not all of them have sufficient buoyancy and water-repellent property that imposes significant limitations in their application. Recently, however, sorbents with acceptable adsorption capacity have been developed based on keratin, produced from pigeon feathers (Zhou et al, 2014). Today, however, commercial adsorbents for removal of oil pollutions from the water surface most often are created on the basis of foamed polymers (polyurethane, polypropylene (Wei et al., 2003), polyethylene, and cross-linked polymers, including rubbers). They are characterized by higher adsorption capacity and buoyancy (Duong et al., 2006). It is noted that natural porous materials and synthetic polymeric foam adsorbents (Duong et al., 2006) demonstrate higher oil sorption at low temperatures or when dealing with a less viscous oil, while in more viscous oil the adsorption capacity decreases because the penetration of the oil into the pores is constrained. Unlike porous adsorbents, fibrous adsorbents such as silk threads and cotton better adsorb viscous oil (Cao and Liu 2006). Since the gaps between the fibers in the fibrous adsorbents are substantially greater than the size of pores in porous sorbents, long-chain molecules at high viscosity do not prevent the sorption, but rather increase the thickness of the oil film on the fibers (Cao and Liu 2006). Despite the fact that oil spills occur during more than half a century, there still is a need for further research in terms of development of improved absorbents at an affordable cost.

Currently in Russia, the absorbents are used insufficiently. As a result, the available absorbents make it possible to collect just 1.5-1.8\% of the total oil spilled to the environment. Despite the large number of research works dealt with the development of absorbents (Adebajo et al., 2003), 
the problem on elimination of oil and oil products pollutions cannot be solved completely so far (Kogan et al., 2014). Existing adsorbents have several drawbacks: they are expensive and some of them have low characteristics. Some of the suggested adsorbents, for example, polymer powders (Dmitrieva et al., 1994) are difficult to use. Other efficient and commercially available sorbents are produced based on exotic raw materials, such as "DULROMABSORB", "Gabsorb-1" and "Gabsorb-2" sorbents, which are made on the basis of a sumauma tree fruits grown on the island of Madagascar (da Conceição, 2005). Currently, quite promising adsorbents of petroleum products, based on graphene materials (MeiJiao et al., 2013; Bi et al., 2014; Lui et al., 2005) as well as adsorbents with magnetic properties (Bachurikhin and Demin, 2008) are under development. However, high production cost hinders their practical implementation. There are also attempts to approach the issues of oil sorption on a comprehensive basis (Kittisrisawai et al., 2015). Therefore, the development of technical solutions for treatment of sewage water and liquidation of emergency oil spills with the use of new, cheaper and more affordable adsorbents is an important and urgent task. In our previous work (Kogan et al., 2014) we have received and reviewed the results obtained during the study of industrial polyurethane foam (PUF) samples. These inexpensive and available organic materials are rigid polyurethane foams used in construction and for cosmetic purposes. Thus, a consideration of factors influencing the process of sorbents synthesis, in particular, polyurethane foam (PUF) that can help clarifying issues related to the processes of their structure formation, remained beyond the scope of our previous research. Study in this direction is necessary for obtaining sorbents with desired set of physical and chemical properties and performance parameters. The aim of this work is to study the effect of temperature on the synthesis of polyurethane as well as to investigate its adsorption characteristics.

\section{Methodology}

The synthesis of industrial PUF was performed out of the Cosmonate M-200 polyisocyanate (Kumno Mitsui Chemicals, Inc., Korea) as a component B, and the saturated polyester "PoliKhim-2001" P-71 (private enterprise
"Khimpostavshchik", Ukraine) - as a component part A at temperatures of $20,30,40,50,60$ and $70^{\circ} \mathrm{C}$ in a cylindrical reactor made of polypropylene, placed in a water bath. The temperature in the reactor was controlled with an accuracy of \pm 1 degree. Equal volumes of components $\mathrm{A}$ and $\mathrm{B}$ were rapidly poured into the reactor and subjected to mixing by submersible mixer. After completion of the reaction, samples of polyurethane foam were cooled in air and then mechanically cleaned against the surface "skin" and crushed to the fraction of 3-8 $\mathrm{mm}$. The methodology for determining the samples buoyancy and their oil absorption capacity as well as the oil used in the current research are identical to those specified in ${ }^{1}$.

To conduct electron microscopic analysis, the samples were coated with platinum in an installation for high vacuum deposition Q150T made by Quorum Technologies (UK). The electron microscopic study was performed on scanning electron microscope Supra 55 VP, of Carl Zeiss (Germany).

\section{RESULTSAND DISCUSSION}

\section{Characteristics of the obtained adsorbents}

All the samples obtained are virtually unsinkable. Desorption phenomenon after samples withdrawal out of oil were not observed. Similarly to the case of industrial PUF, being in a glassy state, the oil absorption kinetic curves of the synthesized PUF (Fig. 1.) are characterized by the presence of maximum (at $30 \mathrm{~min}$ ).

Increase in the synthesis temperature leads to an increase in oil absorption. The PUF production process, in our opinion, can be considered as diffusion-controlled fast physical and chemical process in liquid. The increase of synthesis temperature is accompanied on one hand by a greater and more abrupt gas release of the blowing agent due to the increased diffusion rate in the reaction medium, and, on the other hand, by the increase in interstitial distances in crosslinking of the polymer network due to the increased mobility of large fragments (functional groups) of reagents. Noted circumstance leads to increase in porosity and the fraction of open pores in the PUF structure with increasing synthesis temperature that is confirmed by the electronic microscopic analysis (Fig. 2-4). 
Table 1. The apparent rate of the PUF producing process

\begin{tabular}{|c|c|c|c|c|c|c|}
\hline \multirow{2}{*}{$\begin{array}{l}\text { Kinetic } \\
\text { parameters }\end{array}$} & \multicolumn{6}{|c|}{ Reaction temperature ${ }^{\circ} \mathrm{C}$} \\
\hline & 20 & 30 & 40 & 50 & 60 & 70 \\
\hline $\begin{array}{l}\text { Time of the } \\
\text { process, } s \\
\text { Apparent rate, } \\
10^{3}, \mathrm{~s}^{-1}\end{array}$ & $\begin{array}{l}127 \\
7.87\end{array}$ & $\begin{array}{c}86 \\
11.63\end{array}$ & $\begin{array}{c}67 \\
14.93\end{array}$ & $\begin{array}{c}56 \\
17.86\end{array}$ & $\begin{array}{c}50 \\
20.00\end{array}$ & $\begin{array}{c}46 \\
21.74\end{array}$ \\
\hline
\end{tabular}

The obtained experimental results testify the above mentioned. In particular, while the volume of the resulting PUF sample, synthesized at $20^{\circ} \mathrm{C}$, exceeds the amount of reagents by 14.5 times, in the case of synthesis at a temperature of $70^{\circ} \mathrm{C}$ this figure equals to 18.7 . At that, the density of the obtained samples is reduced by approximately $30 \mathrm{~kg} / \mathrm{m}^{3}$.

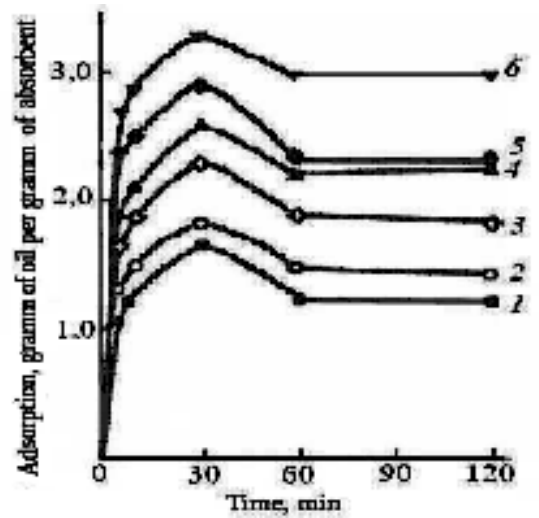

Fig. 1. Oil adsorption curves for PUF adsorbents obtained at the temperatures of 20 (1), $30(2), 40(3), 50(4), 60(5)$ and $70(6){ }^{\circ} \mathrm{C}$

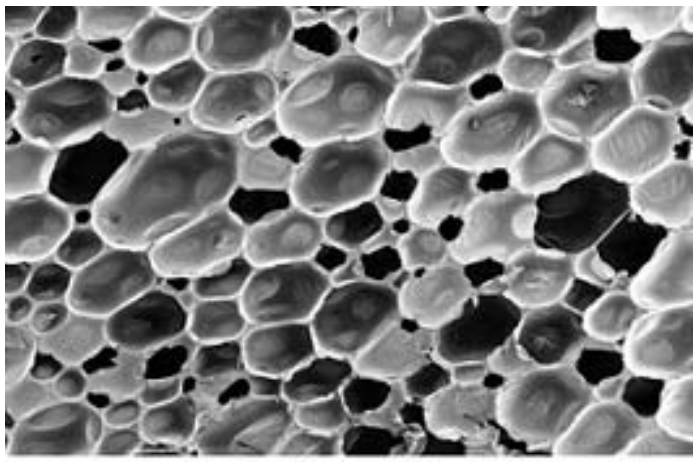

$100 \overrightarrow{\mu m}$

Fig. 3. Electronic micro-photography of the adsorbent surface obtained from the PUF synthesized at $40^{\circ} \mathrm{C}$. Hundredfold magnification
Kinetic regularities of the PUF synthesis process Based on the determination of the process occurrence time $\tau$, when producing PUF at different temperatures, the apparent rate of the process (see Table 1) was calculated by the expression

$$
v_{\text {appar }}=1 / \tau
$$

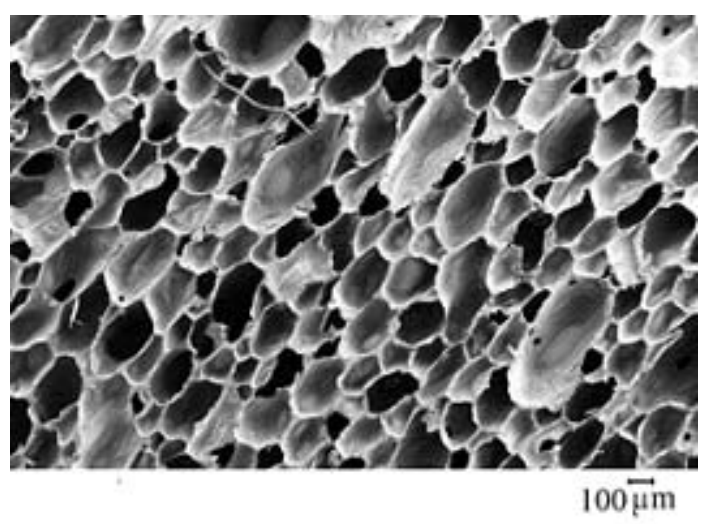

Fig. 2. Electronic micro-photography of the adsorbent surface obtained from the PUF synthesized at $20^{\circ} \mathrm{C}$. Hundredfold magnification

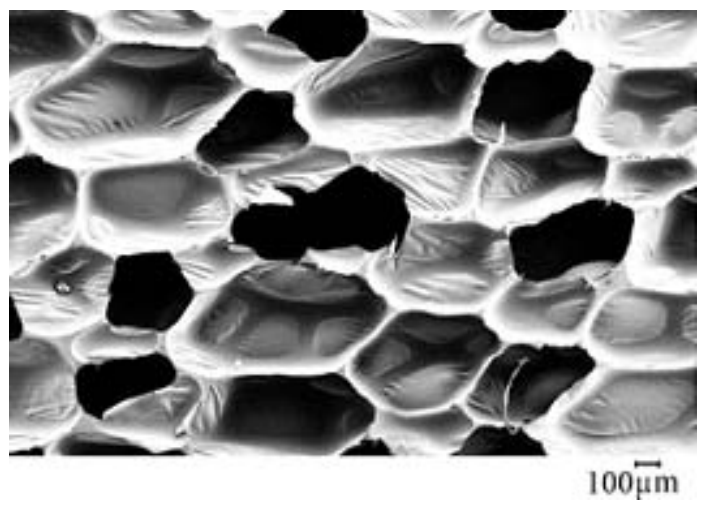

Fig. 4. Electronic micro-photography of the adsorbent surface obtained from the PUF synthesized at $70^{\circ} \mathrm{C}$. Hundredfold magnification 
The kinetic equation in algebraic form for the considered process can be written in the following form

$$
V_{\text {appar }}=k c_{\mathrm{A}}^{x} c_{\mathrm{B}}^{y}
$$

where $k-$ is the effective value that depends at least on the specific reaction rate and the diffusion rate constant of the blowing agent in the reaction volume; $\mathrm{C}_{\mathrm{A}}$ and $\mathrm{C}_{\mathrm{A}}-$ are the molar concentrations of component $\mathrm{A}$ (saturated polyester) and component $B$ (polyisocyanate), relarespectively; $x$ and $y$-are the orders of reaction with respect to components $\mathrm{A}$ and $\mathrm{B}$, respectively. We denote

$$
\begin{array}{r}
\text { than }, c_{\mathrm{A}}^{x} c_{\mathrm{B}}^{y}=B \\
k=v_{\text {appar }} / B
\end{array}
$$

Substituting the obtained expression for $k$ in the Arrhenius equation written in logarithmic form, we get

$$
\ln \frac{V_{\text {appar }}}{B}=\ln A-\frac{E_{a}}{R} \frac{1}{T}
$$

or

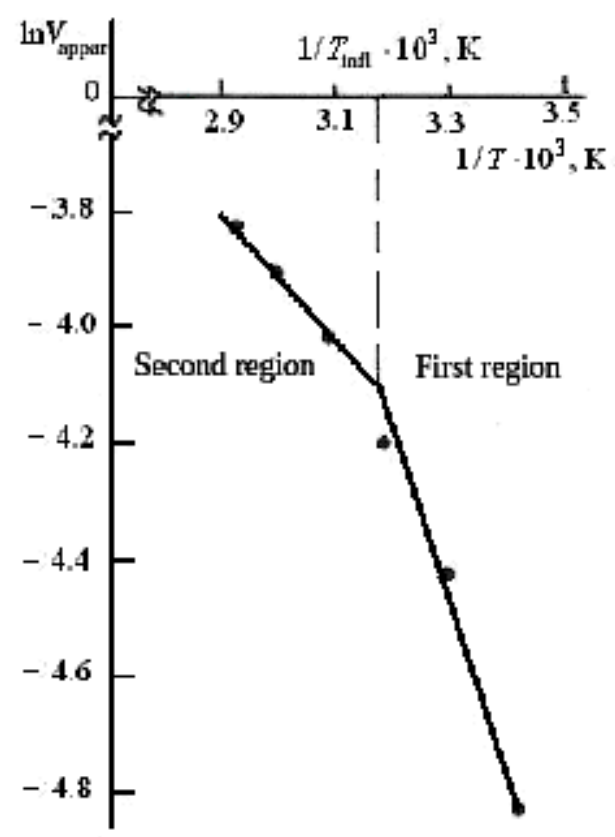

Fig. 5. Dependence of the apparent rate of the PUF synthesizing reaction on the process temperature

$$
\ln V_{\text {appar }}=\ln A+\ln B-\frac{E_{a}}{R} \frac{1}{T}
$$

It is clear that in the case of linear dependence of apparent rate of the process on temperature , the activation energy of the process can be defined graphically as

$$
E_{a}=-R \operatorname{tg} \alpha
$$

The dependence In $V$ appar= $\mathrm{f}(1 / \mathrm{T})$ is shown in Fig. 5. The PUF production is a complex process, consisting of at least the reaction between components $\mathrm{A}$ and $\mathrm{B}$ as well as the diffusion of the blowing agent in the reaction space, ensuring production of foam material. While within the low temperatures range (first region in the figure) the activation energy is $24.94 \mathrm{~kJ} / \mathrm{mole}$, at high temperatures (second region in the figure) it is reduced to $9.15 \mathrm{~kJ} / \mathrm{mol}$. The values of activation energy lie within the range typical for nonequilibrium reactions of polycondensation (polyaddition). The obtained type of dependence is associated most likely with higher diffusion rate and lower activation energy, as well as with decrease of steric hindrance of large fragments $\ln v_{\text {('fü }}=$ ctional $(1 / T)$ groups) of the reagents with increasing temperature above $315.46 \mathrm{~K}$.

At that, it is important to note that this temperature (the temperature of inflection $\mathrm{T}_{\text {infl }}$ ) is comparable with the boiling temperature of the blowing agent - chlorodifluoremethane (313.95 K), i.e. in the second region we have two-phase system consisting of liquid and vapor, while in the first region we have liquid - liquid system.

The obtained experimental results confirm the mentioned above. In particular, while the volume of the resulting PUF sample, synthesized at $20^{\circ} \mathrm{C}$, exceeds the amount of reagents by 14.5 times, in the case of synthesis at a temperature of $70^{\circ} \mathrm{C}$, it exceeds by 18.7 times. At that, the density of the obtained samples is reduced by approximately $30 \mathrm{~kg} / \mathrm{m}^{3}$.

The increase of the PUF synthesis temperature is accompanied by a greater and sharper gas release of blowing agent that is caused by the increase of its diffusion rate in the reaction environment. This is indicated by the results of energy dispersive x-ray microanalysis. Thus, increasing synthesis temperature by $20-40-70^{\circ} \mathrm{C}$, leads to increase of the fluoride content in the PUF 
surface by $0.13-0.46-0.62$ wt. $\%$, respectively, while chlorine - by $1.99-2.80-3.81$ wt.\%, respectively. At that, the only source of fluorine and chlorine in the conducted synthesis of PUF was the blowing agent, i.e. chlorodifluoremethane.

\section{CONCLUSIONS}

1. Regardless of the synthesis temperature, all PUF obtained in the research work as well as industrial PUF that are in a glassy state, are characterized by the availability of the maximum on the oil absorption kinetic curves.

2. Increasing the synthesis temperature leads to an increase in oil absorption that is associated with increasing porosity and the fraction of open pores in the PUF structure.

3. The abovementioned correlates with changes in the PUF volume and density with increasing synthesis temperature that is confirmed by the electron microscopic analysis.

4. The results obtained indicate the future prospects of the research on the impact of the external factors on the PUF-based oil sorbents production process.

In the future we plan to expand our research. In particular, we will continue studying various features of the polyurethane synthesis, including the effect of some additives. Also we expect to carry out experiments on the adsorption of some model hydrocarbons to obtain more rigorous characterization of the process.

\section{REFERENCES}

1. Adebajo, M.O., Frost, R.L., Kloprogge, J.T. Carmody, O., and Kokot, S., "Porous Materials For Oil Spill Cleanup: A Review of Synthesis And Adsorbing Properties," Journal of Porous Materials, 2003; 10(3): 159-170. Retrieved from http://dx.doi.org/10.1023/A:1027484117065. DOI 10.1023/A:1027484117065

2. Annunciado, T.R., Sydenstricker, T.H.D., and Amico S.C., "Experimental Investigation of Various Vegetable Fibers as Sorbent Materials for Oil Spills," Mar. Pollut. Bull., 2005; 50: 1340 1346. DOI:10.1016/j.marpolbul.2005.04.043

3. Bachurikhin, A.L., and Demin, A.V., "The Method of Cleaning the Water Environment against Oil and Oil Products," Patent of the Russian Federation No. RU 2371232; 2008.

4. Bastani, D., Safekordi, A.A., Alihosseini, A., and Taghikhani, V., "Study of Oil Sorption by Expanded Perlite at 298.15K," Sep. Purif. Technol., 2006; 52: 295-306. DOI:10.1016/ j.seppur.2006.05.004

5. Hengchang Bi, Xiao Xie, Kuibo Yin, Yilong Zhou, Shu Wan, Ruoff, R.S. and Tao, L., "Sun Highly Enhanced Performance of Spongy Graphene as Oil Sorbent," J. Mater. Chem. A, 2014; 2: 1652 -1656. DOI: 10.1039/ C3TA14112H

6. Cao, Q., and Liu, P., "Structure and Mechanical Properties of Shape Memory Polyurethane Based on Hyperbranched Polyesters," Polym. Bull., 2006; 57: 889-899. DOI 10.1007/s00289006-0650-z

7. Onuma, C., Frost, R., Xi, Y., and Kokot, S., "Adsorption of Hydrocarbons on Oorganoclays-implications for Oil Spill Remediation," J. Colloid Interface Sci., 2007; 305: 17-24. DOI: $10.1016 /$ j.jcis.2006.09.032

8. Augusto A. da Conceição, "Characteristics of the Tree Named "Samauma" as Potential Natural Resource for the Oil Absorption," Proceedings of the Conf. "Industry. Ecology. Industrial safety”, Ufa. 2005; 51 - 52

9. Augusto A. da Conceição, "Using Natural Absorbent for Repeatedly Collection of Special Diesel Fuel," Proceedings of the Conf. "Industry. Ecology. Industrial safety", Ufa. 2005;

10. Dmitrieva, Z.T., Bondaletov, V.G., Slezka, P.Ya., Kryuchkov, V.I., Merzlikin, A.G., Tereshchenko, I.E., and Getmantsev, V.S., "Method of Adsorption of Crude Oil or Oil Products from Water Surface," Patent of the Russian Federation 1994; ${ }^{1}$ RU 2093640.

11. Duong Hien T. T., and Burford, R. P., "Effect of Foam Density, Oil Viscosity, and Temperature on Oil Sorption Behavior of Polyurethane," J. Appl. Polym. Sci., 2006; 99: 360-367, Retrieved from http://dx.doi.org/10.1002/app.22426. DOI: 10.1002/app.22426.

12. Wei, Q.F., Mather, R.R., Fotheringham, A.F., and Yang, R.D., "Evaluation of Nonwoven Polypropylene Oil Sorbents in Marine Oil-Spill Recovery,” Marine Pollution Bulletin, 2003; 46: 780-783. DOI: 10.1016/S0025-326X(03)000420

13. C. Praba Karan, Rengasamy, R.S., and Dipayan Das, "Oil Spill Cleanup by Structured Fibre Assembly," Indian Journal of Fibre \& Textile Research, 2011; 36(6), pp. 190-200.

14. Kittisrisawai, S., Romero-Zerón, L.B., "Complexation of Surfactant/â-Cyclodextrin to 
Inhibit Surfactant Adsorption onto Sand, Kaolin, and Shale for Applications in Enhanced Oil Recovery Processes. Part I: Static Adsorption Analysis," J. Surfact Deterg., 2015; 18: 603613. DOI $10.1007 / \mathrm{s} 11743-015-1688-4$

15. Tantan Lui, Guizhe Zhao, Wenbui Zhang, Hujuan Chi, Chunlin Hou, and Youyi Sun, "The Preparation of Superhydrophobic Graphene/ Melamine Composite Sponge Applied in Treatment of Oil Pollution," J. Porous Mater, 2005; 22: 1573-1780. DOI 10.1007/s 10934015-0040-8.

16. Lü MeiJiao, Li Jing, Yang XuYu, Zhang ChangAn, Yang Jia, Hu Hao, and Wang Xian Bao, "Applications of Graphene-based Materials in Environmental Protection and Detection," Chinese Science Bulletin. Special issue: Molecular Materials and Devices. 2013; 58(22): 2698-2710. DOI: $10.1007 / \mathrm{s} 11434-013$ - 5887-y

17. Suni, S., Kosunen, A.-L., Hautala, M., Pasila, A., and Romantschuk, M., "Use of a By-product of Peat Excavation, Cotton Grass Fibre, as a Sorbent for Oil-Spills," Mar. Pollut. Bull, 2004; 49: 916-921. DOI:10.1016/j.marpolbul. 2004. 06. 015

18. Kogan, V.E., Zgonnik, P. V., Kovina, D.O., and Chernyaev, V.A., "Foam Glass and Polymer Materials: Effective Oil Sorbents," Glass and Ceramics, 2014; 70(11-12): 425-428. DOI:10.1007/s10717-014-9594-1

19. Long-Tao Zhou, Guang Yang, Xue-Xia Yang, Zhang-Jun Cao, and Mei-Hua Zhou, "Preparation of Regenerated Keratin Sponge from Waste Feathers by a Simple Method and its Potential Use for Oil Adsorption," Environmental Science and Pollution Research, 2014; 21(8): 5730-5736. DOI 10.1007/s11356014-2513-8. 